\title{
Téoros
}

Revue de recherche en tourisme

\section{Le tourisme industriel : à quelles conditions ?}

\section{André Hut}

Volume 15, numéro 2, été 1996

Patrimoine industriel

URI : https://id.erudit.org/iderudit/1075023ar

DOI : https://doi.org/10.7202/1075023ar

Aller au sommaire du numéro

Éditeur(s)

Université du Québec à Montréal

ISSN

0712-8657 (imprimé)

1923-2705 (numérique)

Découvrir la revue

Citer cet article

Hut, A. (1996). Le tourisme industriel : à quelles conditions ? Téoros, 15(2),

19-22. https://doi.org/10.7202/1075023ar d'utilisation que vous pouvez consulter en ligne.

https://apropos.erudit.org/fr/usagers/politique-dutilisation/ 


\section{LE TOURISME INDUSTRIEL: À QUELLES CONDITIONS?}

\section{Andre Hut Belgique}

Un produit touristique nouveau? Une contribution a l'enrichssement culturel et social du visiteur? Une chance de développenent de la région d'accueil? Tour dépend de celai qui prend l'initiative, contrôle l'opération, conçoit et partage l'interprétation.

Le tourisme industriel, sans s'appeler de la sorte, comnenga conne vurisute fechnique puis se restreignit à l'archéologie et au patrimoine industrels avan d'englober les deur, au risque de confusions el de la réduction à un conthun dénonninateur: decouvir un produif et admirer une architecture specifique. l'industrielle?

\section{TOURISME TECHNIQUE}

En 1979, le ministre français de la Jeunesse, des sports et des loisirs, JeanPierre Soisson, met en valeur la nouveauté et l'originalité, si ce n'est l'opportunité, du Guide du Tourisme Technique'. A son avis, celui-ci "comble une lacune d'importance: l'inventaire et la description de réalisations contemporaines issues de la technique et de l'industrialisation qui constituent l'empreinte de notre temps sur le paysage français. Sans prétendre toumer le dos au passé et à ses monuments, cet ouvrage s'adresse à ceux qui apprécient les témoignages des siècles précédents más n'en sont pas moins intéressés par la découverte des sites de notre propre époque[...] montrer aux visiteurs les lieux où l'on travaille, produit, invente, stocke, emmagasine, traite et transforme[...] c cest une participation originale à la promotion du nouveau visage de la France moderne."

L'initiateur et l'auteur, la Société Générale, ajoute que "c"est nussi l'un des rôles d"une grande Banque de faire découvrir el apprécier cette mosaĭque de richesses collectives souvent ignores. Dans un monde maryué d'élonnants progres techniques et scientifiques, l'observalion de l'environtoment economique d'un pays apparâît conne le complément naturel de la connaissince de son histoire, de sa culture, ou de ses coutumes." Dans ce but "de mieux conuaîre... lá France qui produil, exporte et innove"; la couverture arrière du livre précise le contenu. "Plusieurs centaines $(500)$ de réalisations de loule la France: aciéries, raflineries, filatures, verreries d'art ou industrielles, batrages, caves de vins ou de champagne, activiles artisanales, pisciculture, avicullure, arsenaux, sucreties, usines de mécanique, d'électronique, centrales therniques classiques et nucleaires, ports et aérodromes... sont presentêes avec leurs caractéristiques principales, les points fords de la visite et les conditions pratiques..."

En résumé, une viluine des produits et de la technologie française!
A remarquer que le guide reprend également une dizaine de Centres de recherche tournés vers l'avenir comme aussi une cinquantaine de musếes qui représentent déjâ "l'histoire des techniques" (radio, automobile, dentelle, céramique, batellerie, imprimerie, tissus...)

A la même époque d'ailleurs, en 1982, la revue française Esprit s'interroge sur la "Culture technique" dans un numéro spécial et dénonce son ambivalence qui veut que la technique serve le meilleur comme le pire. De plus, l'éditorial éclaire le processus musáal et appelle une réflexion tant sociale qu'éthique. (2)
"Parce que la culture dite technique tait partie de notre environnement immédiat, parce qu'elle réunit à la fois nos gestuelles les plus ordinaires et nos instruments habituels, elle ne Favorise pas la considération. Elle semble le plus souvent impropre lorsqu"elle n"affiche pas le clinquant et le prodige d'un objet «de musée" choisi pour le prestige et l'oubli du reste. Pourtant le temps qui passe, le travail des annees, fortifie les nostal- gies familières, pare certains objets d'une âme, leur extirpe une valeur radicale, plastique, symbolique ou rituelle. Les humains se déshumani- sent, mais les objets nous parlent soudain comme s'ils avaient pris sur eux nos charges pulsionnelles les plus intimes; ce tramway nommé désir... C'est aussi quand on a com- pris que les objets et les machines nous conditionnent qu'on a com- mencé à s'interroger sur eux." 
Quinze ans plus tard, en 1994, une opération "Journée Découverte Entreprises" proposa au grand public de visiter cinquante entreprises. Il s"agissait "de voir et recevoir concrètement, par ceux dont c'est le métier, les réponses à toutes leurs questions. Lesquelles? Sur ha façon dont sont fabriqués les produits que l'on consomme ou que l'on emploie chaque jour" (introduction du responsable du projet, Pascal Deleersyder). Cent mille visiteurs répondaient à l'appel. Un succès.

L"ambe suivante, le Ministre-président de la région wallonne, Robert Collignon, formule, dans l'éditorial de la brochure 1995 , les raisons de son appui. ${ }^{3}$

"Serait-il paradoxe plus difficile à appréhender que celui de voir la Wallonie considérée à travers le monde entier comme une région des plus fertiles, scientifiquement et ćconomiquement parlant, cependant que les Wallons eux-mêmes ignoreraient les richesses qui noutrissent le quotidien de leur région.

Plusieurs études démontrent que la productivité wallonne rejoint les taux les plus élevés du monde. La tradition industrielle de nos contrées n'a d'égale que la qualité des produits présentés et reconnus sur les différents marchés mondiaux, Le savoir-faire des travailleurs et industriels de Wallonie confere à nos entreprises un label utilisé quotidiennement par les attaches commerciaux wallons à travers le monde entier. La Wallonie est présente à travers ses industries et PME, dans les secteurs stratégiques de demain que sont les nouveaux matériaux, les têlécommunications, I'aéronautique, la biotechnologie, l'agro-alimentaire,[...] C'est dans cet esprit, qui est de promouvoir les dynamismes de Wallonie et de ses entreprises, que j'ai accepté avec enthousiasme, l'an dernier, de soutenir la première édition de la journếe "Journée Découverte Entreprises".

C'est pourquoi, cette année, la Région wallonne est d'autant présente aux côtés du secteur industriel dans sa démarche. Cette synergie - je dirais même cette complicité - entre le public et les entreprises de nos régions, est de celles qui génèrent ou accentuent un dynamisme neuf, tout comme y participent de façon quotidienne - exemple des plus fondanentaux = les $s y$ nergies entre nos universilés et le secteur industriel. Les enjeux de cette initiative, faut-il le rappeler, sont multiples.

Il en va en effet d"une vaste opération mettant en relations directes non seulement industries, PME et grand public, mais également tous les acteurs du secteur productif, responsables, chercheurs, travailleurs, clients et fournisseurs. La qualité de nos produits n'est-elle pas le résultat d'une intenction plurielle et positive entre chacun de ces acteurs? Il en va également d"une opération de relations publiques des plus vastes, et qui a pour résultat d'asseoir la notoriété des opérateurs industriels, en Wallonie même tout comme hors de ses frontières.

Il en va enfin de la commassance et de la reconnaissance, par cheun d'entre nous, des effets produits quotidiennement et a tous niveaux pour que la Wallonie puisse perpétuer cette tradition qui est la sienne, d'une terre de culture, de sciences et d'industries."

\section{FERMETURES D'ENTREPRISES}

Entre ces deux discours politiques se sont produites les crises énergétiques et économiques mondiales, les mutations techuologiques en faveur de l'automation et l'informatique qui suppriment beaucoup d'emplois.

De nombreuses entreprises d'extraction (charbon, pierre), de translomation et de services ont ferné leurs portes, mis les ouvitiers et employés au chômage et transformé les tégions jadis prospères des mines, du textile, des verreries ou de lat. métallurgie, en zones économiquement, socialement et environnementalement sinistrées! Telle est d'ailleurs la sil uation au moment où le ministre parle des réussites wallonnes. D'autre part, les entreprises encore en aclivite produisent plus, avec moins de main d $d^{*}$ ceuvre, à moindre coût, grîce à l'intormatisation, mais accunulent une surproduction que les consommateurs appauvris peuvent de moins renouveler et acheter. De plus, des délocalisations de certaines productions dans les filiales du Tiers-Monde provoquent des restructurations de personnel et des licenciements, cette fois dans les cadres et les travailleurs qualifiés, trop onéreux, alors que ces entreprises sont performantes, aboutissant même à des fermetures!

D'où des friches industrielles anciennes ou récentes, des pares industriels qui se vident, des locaux abandonnés, des bureaux inoccupes, souvent vandalisés et squattes.

En définitive, coexistent, dans une dualité dramatique, des entreprises d'avenir, avec des battants, qui réussissent et, en même temps, quelquefois dans les mêmes lieux que celles qui sont mortes, "cimetierres d'emplois"!

\section{PATRIMOINES ET ITINÉRAIRES INDUSTRIELS}

Deux articles ont déjà évoqué et explicité, dans TEOROS ${ }^{4}$ la prise de conscience de la valeur du patrimoine industriel, les inithatives notamment des écomusées, les campagnes des organisations internationales pour une sensibilisation, une réhabilitation et réaffectation de celui-ci, particulièrement par le Conseil de $\mathrm{I}^{\dagger}$ Europe sur le plan éducatif et les subventions de l'Union Européenne sur celui du redéploiement économique.

$\grave{A}$ la demande du premier, dans divers pays du continent, les pouvoirs publics mettent sur pied, en septembre, depuis press de 10 ans, des "Journées du Paurimoine". Dans la région wallonne de Belgique, la "Division du Patrimoine" de l'administration du territoire avait choisi, comme theme mobilisateur, en 1994 , le paluimoine industriel et favorisé la découverte, par un grand public, des initiatives animées par les associations locales dans des lieux pas necessairement touristiques, au contraire.

Après cet événement, deux associations Ic P,I,W, B. (Patrimoine industriel Wallonie-Bruxelles) el ICOMOS (International Council of Monuments and Sites) de Wallonie-Bruxelles, avec le soutien de l'administration mentionnée, ont organisé un séminaire intitulé: "Les journées du Patrimoine (industriel). Et apres? ?" Cinq tables rondes ont tenté de 
répondre à la question dans le but que ce mouvement amorces continue. Outre les problemes de protection et de conservation, de restauration et de réaffectation, de mise en valeur par la promotion et la gestion, le demier sujet abordé rejoint directement les préoccupations de cet article: "Des itineraires pour une sensibilisation au patrimoine industriel". Quelques extraits du texte introductif au débat par celui qui exerce déjà ce genre de pratique avec son association, la Fonderie, Guido Vanderhulst:

"Organiser des itineraires de sensibilisation au patrimoine industriel dans la ville et la campagne, dans les faubourgs et les banlieues, ne peut se limiter à decrire des immeubles et leur architecture.

Il $\mathrm{s}^{7}$ agit en effet $\mathrm{d}^{7}$ apprendre à lire les murs souvent noirs et lépreux d'anciennes banlieues où le touriste n'a pas sa place, ou parfois mijestueux et superbes sur certains sites industriels, pour y rappeler le geste anonyme de milliers de femmes et d'hommes qui ont fait et font encore de leurs mains ces mille choses de notre quotidien, du boire et du manger, de l'habillement aux outils, à l'habitat, aux meubles, aux déplacements, à la communication.

L'itinéraire industriel expliquera la façon dont les industries ont conçu, payé et voulu l'implantation de certains complexes et immeubles, avec une volonté manifeste de contrôler le circuit de production, les coûts de production et la rentabilice du traviil de l'homme. II s'agit d'y voir l'apport des architectes et des modes, celui des ingénieurs et de l'évolution des techniques de construction, et les astuces des ouvriers eux-mêmes pour se faciliter la vie au travail.

Il s'agit encore d'expliquer et souligner l'implantation des industries et le lien qu'il y a entre elles, l'impact et les modifications apportées au paysage, l'articulation des fonctions urbaines, du logement des ouvriers, de leurs patrons et contremaîtres, aux entrepôts, aux tracés des canaux, des voies de chemins de fer et des routes.

Il s'agit aussi d'expliquer comment les procédes de fabrication fonctionnent, comment l'usine ou l'atelier sont, en quelque sorte, construils autour de ces produits, comment et où sont situés les ouvières et ouvriers et dans quelles conditions ils travaillent, comment et où ils revendiquent améliorations et dignité et pourquoi."

De tout cela, ${ }^{4}$ le bâtiment industriel est la trace, le cadre, le milieu, le creuset". (5) Et le présentateur de proposer, notanment, deux questions auxquelles il invite les participants de la table ronde de répondre. " $\mathrm{D}^{\dagger}$ abord, pourquoi celte mode du "patrimoine industriel" quand l'industrie ferme, se restructure et se délocalise? Nest-ce pas un jeu malsain? Ensuite, patrimoine industriel et circuit de valorisation: qui le définit? selon quels criteres? Architecturaux, techniques, sociaux, affectifs, scientiliques ou techniques?"

\section{TOURISME INDUSTRIEL}

C' est pourquoi on a le droil de s'inquicter si les entreprises touristiques commencent à sintéresser à celui-ci. Dans une collection qui tépond â un objectif de tormation génétale et professionnelle chapitte consacré au tourisme culturel ne manque pas, c'est nouveau et posilif, de donner une place égale au tourisme industricl par lapport aux autres qu'ils soient historique, religicux, viticole on gastronomique ainsi que d'aflinmer que les "lieux ef objets industriels sont actuellement valorisés comme appartenant au patrimoine culturel". Cependiant, l'auteur formule limitativement son approthe exclusivement en termes de "sites et bâtiments" que l'on peut visiter: ceux de l'ancien i'tégine, de la Révolution industriclle du XIXe siccle ou enlin ceuX du $X X e$ siecle.

Mais patadoxalement, les siles actuels ne reprennent pas los entreprises du "Tourisme technique" mentionnes plas haut mis se cantomnent seulement ha l'une des catégories notanment "les barrages en montagne, centrales hydroćlecuriques ou nucléaires, les grands ponts et aéroports futuristes qui participent eux aussi, dil-il, au palrinoine industriel," Complémentairement, l'auleur ne s'interesse qu" $\mathrm{a} \mathrm{l}^{\prime a t}$ atilecture industrielle des batiments of ne lail atucune allusion a leurs dimensions economiques, rechniques, sociales ou coviontementates.
Le risque est grand que les guides touristiques - souvent historiens de l'art ou formés par eux - accompagnant les groupes de visiteurs, ne se contentent que de cette seule approche architecturale et esthetisante comme ils le font d'ailleurs malheureusement pour le patrimoine religieux.

Guido Vanderhulst a raison de s'interroger sur les compétences du guide dans ce domaine particulier.

"C.est donc que le guide chargé de pareils itinéraires n'est pas n' importe qui. Quelles exigences doit-on lui imposer et quelle sera sa formation? Est-il seul ou porte-parole d"une Equipe de recherche? Il doit devenir, bien plus que sur d'autres itinêraires, communicateur d'une histoire de petites gens, mais aussi des techniques, et d'un vécu d"une complexité peu commune. Si la passion pour les hommes $n^{4} y$ est pas plus que pour l'architecture, de quel patrimoine industriel parlera le guide?[... $]^{s}$

[...] En effet, la spécificité des guides du patrimoine industriel réside notamment dans la pluridisciplinarité des connaissances: historiques, sociales, techniques, connaissances du patrimoine [... ]

La matiere des guides du patrimoine industriel étant ancrée plus que tout autre dans la vie, il faut être attentif a ne pas se réfugier derriere la neutralité technique pour oblitérer le social. Qui serait le guide ideal? Ce guide serait-il le mineur, le chef instructeur d'une equipe de travailleurs, le guide formé theoriquement et faisant une synthèse?[...]

[...] Un encadrement scientifique avec verification est délinitivement indispensable. $N^{2}$ oublions pas que la formation de la releve est une queslion majeure en soi." "

L'autre danger réside dans le fait de dépossóder les habitants et travailleurs de ces entreprises, du présent et du passé, de leur identité, leur histoire, leur vécu, leurs aspiralions sociales, leur culture propre! Pour le passé, ajoute le directeur de la Fonderie:

"[...] ces immeubles et ces sites sont patrimoine d'une population qui parfois s'y est identifíce et dont la pous- 
sière colle encore à la peat. Cente population a souvent vécu la fermeture des ateliers et industries comme un échec et une exclusion: c'est pourtant a elle de devenir les principaux vecteurs et acteurs de l'histoire racontée aux générations à venir. Le guide doit s'en persuader et rendre l'histoire qu"il a apprise à ceux qui l'ont vécue pour apprendre à son tour." s

C'est la raison pour laquelle il met en cause les autorités en leur demandant

\begin{abstract}
"comment faire pour que le palrimoine industriel ne se réduise pas à quelques belles architectures alors que bien des immeubles et sites n'ont de qualité que le tếmoignage ei d'avoir étế le creuset d'une longue et parfois tres pénible histoire des hommes? Comment présenter lat Culture industrielle comme une culture aussi?"
\end{abstract}

Pour le présent, les organisateurs et animateurs des mouvements d'éducation populaire, qui bâtissent des programmes d'excursions pour leurs membres, doivent Savoir que la personne désignée pour guider la visite dans l'entreprise, souvent cadre ou technicien, s"en liendra atux explications techniques de la fabrication ou du fonctionnement concernant le produit, ne relevant éventuellement le sawoirfaire des travailleurs que comme une plus-value qualitative de celui-ci.

Ne faudrait-il pas prévoir, en dehors de l'entreprise, dans un local voisin, de rencontrer, awant ou après la visite, des personnes qui puissent apporter des informations complémentaires sur l'historique do l'entreprise, les conditions de travail, les luttes revendicatrices, les conséquences humaines el sociales du traviail sur la santé, le temps libre, la retraite ellemême. En fait des tếmoins: retraité, ancien travailleur de l'entreprise ou l'un de ceux-ci, après ou avant son travail!

En conséquence, si pour les sociologues du tourisme, le tourisme industriel englobe aussi bien les entreprises du présent que celles du passć. pourguoi ne pas en tirer parti pédagogiguement, diuns un processus d'animation touristique, en tissant des liens, inlerdépendants et complémentaires, entre la recherche scicn- tifique fondamentale, en amont, les applications techniques, en aval, dans les entreprises actuelles, l'êvolution historique de celles-ci dans les musées ad hoc, les représentations symboliques impliquées dans la publicité et destinées à influencer l'imaginaire en vue d'acculer à l'achat du produit?

A orchestrer autour de filières - bois, pierre, or, boissons, pain, textile - ou de thématiques: l'eau, le fleuve, la ville, le livre ou le journal, les décheis; de l'écosystème aux divers utilisateurs et responsables professionnels ou politiques ainsi qu'aux rẹrésentalions (vidéos, photos, peintures...) ou aux récits et histoires de vie de témoins, y compris dans la presse!

Les dés sont particulièrement pipés quand $c^{*}$ est l'entreprise elle-mêtme qui crée son propre musée comme Coca-Cola à Allanta, le chocolat Jacques en Belgique ou le nouveau parc à thème, en Allemagne, de Warner Brothers let assez logiquement appelé "Warner Brothers Movie World" (sic)]

Ne fiudrait-il pas impliquer également, d'une part les associations de consommateurs qui analysent, en avald, la qualitếprix du produit "mis en valleur", et, d'autre part. Ies ONG qui peuvent restituer, en amont, les origines des matières premières utilisees, les manipulations internationales de leur prix d'achat - par exemple, le cacao - l'exploilation des populations autochtones dans leur production, notamment les cnfants, la prếparation avant le départ dans le 'liers-Monde - par exemple, à Cubit ou au Mexique - et l'accueil, à l'arrives, en laveur d"un tourisme intelligent conscicnisulcur?

Les écologistes ont une place privilégiéc pour expliciler les conséquences néfatistes des luméces, des déchols, des rejets d"eaux usếes douns l'environnement do l'entreprise, notamment â partir des séquelles du passé, repérables dans le patrimoine industriel, manifeste dans les pays de I'Est de l'Europe.

Les divers risques ef dangers comme aussi les chances éducatives interpellent les associatlions citces allin de donner atu tourisme industriel la possibilite de contribuer au développement economiçue. social et culturel tant des populations locales qui l'ont reçu en héritage et en charge qu' aux visiteurs afin qu'ils prennent conscience des enjeux humains et spirituels qui sont en cause.

Les partenariats entre associations sont indispensables, les collaborations transversales et les décloisonnements aussi. La solidarité des travailleurs et leurs luttes tracent la voie à suivre.

\section{RÉFÉRENCES}

1 Guide Société Générale du Tourisme technique, Edition SOLAR - 1979.

2 Yan de Kerorguen. *Vous avez dit Culture technique?s, in Esprit, Paris, octobre 1982.

3. Brochure "Journées Découverte Entreprises". Dimanche 1er octobre 1995.

4 Andre Hut. wLe Tourisme patrimonial, l'experrience belge" et "Eur-Info" sur les actions internationales en faveur de l'interaction environnement et tourismes, in TEOROS. Vol. 10, no 1, mars 1991.

5 Journécs du Patrimoine et après? Actes du séminaire et PIWB-ICOMOS. Numéro spécial du bulletin trimestriel du PIWB, no 32. Septenbre 1995 - Musée d'Ames, Quai de Maestrichi, 8 - 4000 Liège (Tel.: 041219416 - FAX: 041.2194.01). Les citations tirtes de ce document se trouvent aux pages $55,56,57$ et 58 .

6 Claude Peyroutet. La France touristique. Paris, Eld. Nathan, coll. «Reperes pratiquesw, 1995 , 